Article

\title{
Some Estimates for Generalized Riemann-Liouville Fractional Integrals of Exponentially Convex Functions and Their Applications
}

\author{
Saima Rashid $^{1}$, Thabet Abdeljawad ${ }^{2, * \mathbb{D}}$, Fahd Jarad ${ }^{3}$ and Muhammad Aslam Noor $^{1}$ (D) \\ 1 Department of Mathematics, COMSATS University Islamabad, Islamabad 45550, Pakistan \\ 2 Department of Mathematics and General Sciences, Prince Sultan University, P.O. Box 66833, \\ Riyadh 11586, Saudi Arabia \\ 3 Department of Mathematics, Çankaya University, Etimesgut, 06790 Ankara, Turkey \\ * Correspondence: tabdeljawad@psu.edu.sa
}

Received: 29 July 2019; Accepted: 20 August 2019; Published: 2 September 2019

\begin{abstract}
In the present paper, we investigate some Hermite-Hadamard $(\mathcal{H} \mathcal{H})$ inequalities related

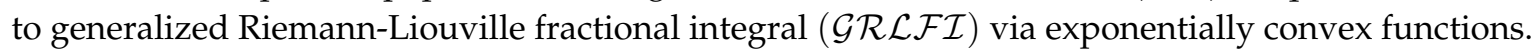
We also show the fundamental identity for $\mathcal{G} \mathcal{R} \mathcal{L} \mathcal{F}$ having the first order derivative of a given exponentially convex function. Monotonicity and exponentially convexity of functions are used with some traditional and forthright inequalities. In the application part, we give examples and new inequalities for the special means.
\end{abstract}

Keywords: convex function; exponentially convex function; fractional integrals; generalized Riemann-liouville fractional integrals

\section{Introduction}

Recently, several researchers have attracted the fractional calculus, see References [1-4]. The effect and motivation of this fractional calculus in both theoretical and applied science and engineering rose substantially. Numerous studies related to the discrete versions of this fractional calculus have been established, which benefit from countless applications in the theory of time scales, physics, different fields of engineering, chemistry and so forth (e.g, see References [4-32] and the references therein).

A few decades ago, a lot of new operator definitions were given and the properties and structures of these operators have been examined. Some of these operators are very close to classical operators in terms of their characteristics and definitions. It is known that the $\mathcal{G} \mathcal{R} \mathcal{L} \mathcal{F} \mathcal{I}$, which was introduced in reference [33], extends several well-known fractional integral operators (see Remark 1 below). Both the generalized Riemann-Liouville fractional derivative and the integral operator are useful in the study of transform theory, quantum theory and fractional intgerodifferential equations.

Almost every mathematician knows the importance of convexity theory in every field of mathematics, for example in nonlinear programming and optimization theory. By using the concept of convexity, several integral inequalities have been introduced such as Jensen, $\mathcal{H} \mathcal{H}$ and Slater inequalities, and so forth. But the well-known one is the celebrated $\mathcal{H} \mathcal{H}$ inequality.

Let $\mathcal{I} \subseteq \mathcal{R}$ be an interval and $\mathcal{U}: \mathcal{I} \rightarrow \mathcal{R}$ be a convex function. Then the double inequality

$$
\mathcal{U}\left(\frac{d_{1}+d_{2}}{2}\right) \leq \frac{1}{d_{2}-d_{1}} \int_{d_{1}}^{d_{2}} \mathcal{U}(x) d x \leq \frac{\mathcal{U}\left(d_{1}\right)+\mathcal{U}\left(d_{2}\right)}{2}
$$


holds for all $d_{1}, d_{2} \in \mathcal{I}$ with $d_{1}<d_{2}$. It is easy to see that if $\mathcal{U}$ is concave on $\mathcal{I}$, one has the reverse of this inequality. This inequality provides bounds for the mean value of a convex function. Recently, mathematicians have focused on obtaining new variants of the $\mathcal{H} \mathcal{H}$ inequality by giving generalizations, improvements, refinements and extensions, see References [34-36].

Exponentially convex functions have emerged as a significant new class of convex functions, which have important applications in technology, data science and statistics. The main motivation of this paper depends on new inequalities that have been proved via $\mathcal{G R \mathcal { L F }}$ and applied for exponentially convex functions. This identity offers new upper bounds and estimations of $\mathcal{H} \mathcal{H}$ type integral inequalities. Some particular cases have been discussed, which can be deduced from these consequences.

Recall the definition of an exponentially convex function, which is investigated by Dragomir and Gomm [34]:

Definition 1. (See [34]) A positive real-valued function $\mathcal{U}: K \subseteq \mathcal{R} \longrightarrow(0, \infty)$ is said to be exponentially convex on $\mathrm{K}$ if the inequality

$$
e^{\mathcal{U}(\tau x+(1-\tau) y)} \leq \tau e^{\mathcal{U}(x)}+(1-\tau) e^{\mathcal{U}(y)}
$$

holds for $x, y \in K$ and $\tau \in[0,1]$.

Exponentially convex functions are used to manipulate for statistical learning, sequential prediction and stochastic optimization, see References [37-39].

After the class of exponentially convex functions was introduced by Dragomir and Gomm [34], Alirezai and Mathar [37] have investigated the mathematical perspectives along with their fertile applications in statistics and information theory, see References [37,39]. Due to its significance, Pecaric and Jaksetic [40,41] used another kind of exponentially convex function introduced in Reference [42] and have provided some applications in Euler-Radau expansions and stolarsky means. Our intention is to use the exponentially convexity property of the functions as well as the absolute values of their derivatives in order to establish estimates for $\mathcal{G} \mathcal{R} \mathcal{L} \mathcal{F} \mathcal{I}$.

Definition 2. ([1-3]) Let $\left(d_{1}, d_{2}\right)\left(-\infty \leq d_{1}<d_{2} \leq \infty\right)$ be a finite or infinite real interval and $\rho>0$. Let $\Psi(x)$ be an increasing and positive monotone function on $\left(d_{1}, d_{2}\right]$ with a continuous derivative on $\left(d_{1}, d_{2}\right)$. Then the left and right-sided generalized Riemann-Liouville fractional integrals of a function $\mathcal{U}$ on $\left[d_{1}, d_{2}\right]$ are defined by

$$
I_{d_{1}^{+}}^{\rho, \Psi} \mathcal{U}(\tau)=\frac{1}{\Gamma(\rho)} \int_{d_{1}}^{\tau} \Psi^{\prime}(x)(\Psi(\tau)-\Psi(x))^{\rho-1} \mathcal{U}(x) d x,
$$

and

$$
I_{d_{2}^{-}}^{\rho, \Psi} \mathcal{U}(\tau)=\frac{1}{\Gamma(\rho)} \int_{\tau}^{d_{2}} \Psi^{\prime}(x)(\Psi(x)-\Psi(\tau))^{\rho-1} \mathcal{U}(x) d x,
$$

respectively; with $\Gamma($.$) , the classical gamma function.$

Remark 1. Many known defined fractional integral operators are just special cases of (2) and (3).

1. Setting $\mathcal{U}(\tau)=\tau$, it turns into the both sided (RLI).

2. Setting $\mathcal{U}(\tau)=\log \tau$, the Hadamard fractional integrals are obtained [1,3].

3. Setting $\mathcal{U}(\tau)=\frac{\tau^{\beta}}{\beta}, \beta>0$ it turns into the both sided Katugampola fractional integrals given in Reference [33].

4. Setting $\mathcal{U}(\tau)=\frac{(\tau-a)^{\beta}}{\beta}, \beta>0$, the operators in reference [43] are obtained.

5. Setting $\mathcal{U}(\tau)=\frac{\tau^{\epsilon+\varsigma}}{\epsilon+\zeta}$, it turns into the both sided generalized conformable fractional integrals defined by Khan et al. in reference [44]. 
The principal objective of this paper is to use a new convex class and a new integral operator to obtain new versions of $\mathcal{H} \mathcal{H}$-inequality that give bounds for the mean value of convexity. Also, we will establish some more general estimates and related modulus inequalities for $\mathcal{G} \mathcal{R} \mathcal{L} \mathcal{F} \mathcal{I}$ via exponentially convex functions. In addition, the accuracy of the results was tested with applications of special means and with some examples.

\section{2. $\mathcal{H} \mathcal{H}$ Inequality for $\mathcal{G} \mathcal{R} \mathcal{L} \mathcal{F} \mathcal{I}$}

Theorem 1. Let $\mathcal{U}:\left[d_{1}, d_{2}\right] \rightarrow \mathcal{R}$ be a positive function, for $0 \leq d_{1}<d_{2}$, and $e^{\mathcal{U}} \in L_{1}\left(\left[d_{1}, d_{2}\right]\right)$. Let $\Psi(x)$ is an increasing and positive monotone function on $\left(d_{1}, d_{2}\right]$, with continuous derivative $\Psi^{\prime}(x)$ on $\left(d_{1}, d_{2}\right)$. Let $\mathcal{U}$ is an exponentially convex function and $\rho \in(0,1)$. Then

$$
e^{\mathcal{U}\left(\frac{d_{1}+d_{2}}{2}\right)} \leq \frac{\Gamma(\rho+1)}{\left(d_{2}-d_{1}\right)^{\rho}}\left[I_{d_{1}^{+}}^{\rho, \Psi}\left(e^{\mathcal{U}} \circ \Psi\right)\left(\Psi^{-1}\left(d_{2}\right)\right)+I_{d_{2}^{-}}^{\rho, \Psi}\left(e^{\mathcal{U}} \circ \Psi\right)\left(\Psi^{-1}\left(d_{1}\right)\right)\right] \leq \frac{e^{\mathcal{U}\left(d_{1}\right)}+e^{\mathcal{U}\left(d_{2}\right)}}{2} .
$$

Proof. Since $\mathcal{U}$ is an exponentially convex function for $\left[d_{1}, d_{2}\right]$, we have

$$
e^{\mathcal{U}\left(\frac{u+v}{2}\right)} \leq \frac{e^{\mathcal{U}(u)}+e^{\mathcal{U}(v)}}{2} .
$$

Let $u=\tau d_{1}+(1-\tau) d_{2}$ and $v=(1-\tau) d_{1}+\tau d_{2}$, we get

$$
2 e^{\mathcal{U}\left(\frac{d_{1}+d_{2}}{2}\right)} \leq e^{\mathcal{U}\left(\tau d_{1}+(1-\tau) d_{2}\right)}+e^{\mathcal{U}\left((1-\tau) d_{1}+\tau d_{2}\right)} .
$$

Multiplying by $\tau^{\rho-1}$ on both sides of inequality (6) and then integrating w.r.t $\tau$ over $[0,1]$, implies

$$
\frac{2}{\rho} e^{\mathcal{U}\left(\frac{d_{1}+d_{2}}{2}\right)} \leq \int_{0}^{1} \tau^{\rho-1} e^{\mathcal{U}\left(\tau d_{1}+(1-\tau) d_{2}\right)} d \tau+\int_{0}^{1} \tau^{\rho-1} e^{\mathcal{U}\left((1-\tau) d_{1}+\tau d_{2}\right)} d \tau .
$$

Now consider,

$$
\begin{aligned}
& \frac{\Gamma(\rho+1)}{2\left(d_{2}-d_{1}\right)^{\rho}}\left[I_{d_{1}^{+}}^{\rho, \Psi}\left(e^{\mathcal{U}} \circ \Psi\right)\left(\Psi^{-1}\left(d_{2}\right)\right)+I_{d_{2}^{-}}^{\rho, \Psi}\left(e^{\mathcal{U}} \circ \Psi\right)\left(\Psi^{-1}\left(d_{1}\right)\right)\right] \\
& =\frac{\Gamma(\rho+1)}{2\left(d_{2}-d_{1}\right)^{\rho}} \frac{1}{\Gamma(\rho)}\left[\int_{\Psi-1\left(d_{1}\right)}^{\Psi^{-1}\left(d_{2}\right)} \Psi^{\prime}(z)\left(d_{2}-\Psi(z)\right)^{\rho-1}\left(e^{\mathcal{U}} \circ \Psi\right)(z) d z\right. \\
& \left.+\int_{\Psi^{-1}\left(d_{1}\right)}^{\Psi^{-1}\left(d_{2}\right)} \Psi^{\prime}(z)\left(\Psi(z)-d_{1}\right)^{\rho-1}\left(e^{\mathcal{U}} \circ \Psi\right)(z) d z\right]
\end{aligned}
$$

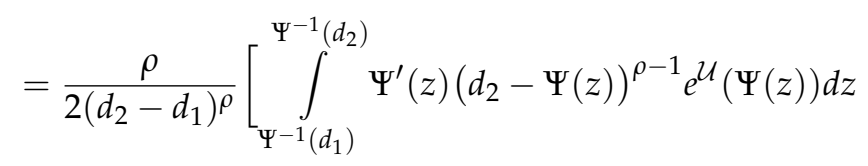

$$
\begin{aligned}
& \left.+\int_{\Psi^{-1}\left(d_{1}\right)}^{\Psi^{-1}\left(d_{2}\right)} \Psi^{\prime}(z)\left(\Psi(z)-d_{1}\right)^{\rho-1} e^{\mathcal{U}}(\Psi(z)) d z\right] \\
& =\frac{\rho}{2}\left[\int_{0}^{1} \tau^{\rho-1} e^{\mathcal{U}\left(\tau d_{1}+(1-\tau) d_{2}\right)} d \tau+\int_{0}^{1} \tau^{\rho-1} e^{\mathcal{U}\left((1-\tau) d_{1}+\tau d_{2}\right)} d \tau\right] \\
& \geq e^{\mathcal{U}\left(\frac{d_{1}+d_{2}}{2}\right)} \text {, }
\end{aligned}
$$


by using (7). Thus the first inequality of (4) is proved.

Next, we again use the exponential convexity of $\mathcal{U}$, that is,

$$
e^{\mathcal{U}\left(\tau d_{1}+(1-\tau) d_{2}\right)}+e^{\mathcal{U}\left((1-\tau) d_{1}+\tau d_{2}\right)} \leq e^{\mathcal{U}\left(d_{1}\right)}+e^{\mathcal{U}\left(d_{2}\right)} .
$$

Multiplying by $\tau^{\rho-1}$ on both sides of inequality (8), and then integrating w.r.t $\tau$ over $[0,1]$, implies

$$
\int_{0}^{1} \tau^{\rho-1} e^{\mathcal{U}\left(\tau d_{1}+(1-\tau) d_{2}\right)} d \tau+\int_{0}^{1} \tau^{\rho-1} e^{\mathcal{U}\left((1-\tau) d_{1}+\tau d_{2}\right)} d \tau \leq \frac{e^{\mathcal{U}\left(d_{1}\right)}+e^{\mathcal{U}\left(d_{2}\right)}}{\rho} .
$$

That is,

$$
\frac{\Gamma(\rho+1)}{\left(d_{2}-d_{1}\right)^{\rho}}\left[I_{d_{1}^{\rho}}^{\rho \Psi}\left(e^{\mathcal{U}} \circ \Psi\right)\left(\Psi^{-1}\left(d_{2}\right)\right)+I_{d_{2}^{-}}^{\rho, \Psi}\left(e^{\mathcal{U}} \circ \Psi\right)\left(\Psi^{-1}\left(d_{1}\right)\right)\right] \leq \frac{e^{\mathcal{U}\left(d_{1}\right)}+e^{\mathcal{U}\left(d_{2}\right)}}{2} .
$$

Hence the proof is completed.

Our next result is the subsequent lemma, which is useful for our coming results.

Lemma 1. Let $\mathcal{U}:\left[d_{1}, d_{2}\right] \rightarrow \mathcal{R}$ be a differentiable mapping, for $0 \leq d_{1}<d_{2}$, and e $\mathcal{U} \in L_{1}\left(\left[d_{1}, d_{2}\right]\right)$. Let $\Psi(x)$ is an increasing and positive monotone function on $\left(d_{1}, d_{2}\right]$, with continuous derivative $\Psi^{\prime}(x)$ on $\left(d_{1}, d_{2}\right)$ and $\rho \in(0,1)$. Then

$$
\begin{aligned}
& \frac{e^{\mathcal{U}\left(d_{1}\right)}+e^{\mathcal{U}\left(d_{2}\right)}}{2}-\frac{\Gamma(\rho+1)}{2\left(d_{2}-d_{1}\right)^{\rho}}\left[I_{d_{1}^{\rho}}^{\rho, \Psi}\left(e^{\mathcal{U}} \circ \Psi\right)\left(\Psi^{-1}\left(d_{2}\right)\right)+I_{d_{2}^{-}}^{\rho, \Psi}\left(e^{\mathcal{U}} \circ \Psi\right)\left(\Psi^{-1}\left(d_{1}\right)\right)\right] \\
& =\frac{1}{2}\left[\int_{\Psi^{-1}\left(d_{1}\right)}^{\Psi^{-1}\left(d_{2}\right)} \Psi^{\prime}(z)\left(\frac{\Psi(z)-d_{1}}{d_{2}-d_{1}}\right)^{\rho}\left(e^{\mathcal{U}} \circ \Psi\right)(z) \mathcal{U}^{\prime}(\Psi(z)) d z\right. \\
& \left.-\int_{\Psi^{-1}\left(d_{1}\right)}^{\Psi^{-1}\left(d_{2}\right)} \Psi^{\prime}(z)\left(\frac{d_{2}-\Psi(z)}{d_{2}-d_{1}}\right)^{\rho}\left(e^{\mathcal{U}} \circ \Psi\right)(z) \mathcal{U}^{\prime}(\Psi(z)) d z\right] .
\end{aligned}
$$

Proof. Consider

$$
\begin{aligned}
\mathbb{I}= & \frac{d_{2}-d_{1}}{2} \int_{0}^{1}\left[(1-\tau)^{\rho}-\tau^{\rho}\right] e^{\mathcal{U}\left((1-\tau) d_{1}+\tau d_{2}\right)} \mathcal{U}^{\prime}\left((1-\tau) d_{1}+\tau d_{2}\right) d \tau \\
= & \frac{d_{2}-d_{1}}{2}\left[\int_{0}^{1}(1-\tau)^{\rho} e^{\mathcal{U}\left((1-\tau) d_{1}+\tau d_{2}\right)} \mathcal{U}^{\prime}\left((1-\tau) d_{1}+\tau d_{2}\right) d \tau\right. \\
& \left.-\int_{0}^{1} \tau^{\rho} e^{\mathcal{U}\left((1-\tau) d_{1}+\tau d_{2}\right)} \mathcal{U}^{\prime}\left((1-\tau) d_{1}+\tau d_{2}\right) d \tau\right] .
\end{aligned}
$$

By making a change of variable in the above equation $\Psi(z)=(1-\tau) d_{1}+\tau d_{2}$, we have 


$$
\begin{aligned}
\mathbb{I}= & \frac{1}{2} \int_{\Psi^{-1}\left(d_{1}\right)}^{\Psi^{-1}\left(d_{2}\right)} \Psi^{\prime}(z)\left(\frac{d_{2}-\Psi(z)}{d_{2}-d_{1}}\right)^{\rho}\left(e^{\mathcal{U}} \circ \Psi\right)(z) \mathcal{U}^{\prime}(\Psi(z)) d z \\
& \left.-\int_{\Psi^{-1}\left(d_{1}\right)}^{\Psi^{-1}\left(d_{2}\right)} \Psi^{\prime}(z)\left(\frac{\Psi(z)-d_{1}}{d_{2}-d_{1}}\right)^{\rho}\left(e^{\mathcal{U}} \circ \Psi\right)(z) \mathcal{U}^{\prime}(\Psi(z)) d z\right] \\
= & I_{1}+I_{2} .
\end{aligned}
$$

Now

$$
\begin{aligned}
& \frac{\Gamma(\rho+1)}{2\left(d_{2}-d_{1}\right)^{\rho}} I_{d_{1}^{+}}^{\rho, \Psi}\left(e^{\mathcal{U}} \circ \Psi\right)\left(\Psi^{-1}\left(d_{2}\right)\right) \\
& =\frac{\rho}{2\left(d_{2}-d_{1}\right)^{\rho}} \int_{\psi^{-1}\left(d_{1}\right)}^{\psi^{-1}\left(d_{2}\right)} \psi^{\prime}(z)\left(d_{2}-\psi(z)\right)^{\rho-1}\left(e^{\mathcal{U}} \circ \psi\right)(z) d z \\
& =-\frac{1}{2\left(d_{2}-d_{1}\right)^{\rho}} \int_{\psi^{-1}\left(d_{1}\right)}^{\psi^{-1}\left(d_{2}\right)}\left(e^{\mathcal{U}} \circ \psi\right) d\left(d_{2}-\psi(z)\right)^{\rho} \\
& =\frac{1}{2\left(d_{2}-d_{1}\right)^{\rho}}\left[\left(d_{2}-d_{1}\right)^{\rho} e^{\mathcal{U}\left(d_{1}\right)}+\int_{\psi^{-1}\left(d_{1}\right)}^{\psi^{-1}\left(d_{2}\right)} \psi^{\prime}(z)\left(d_{2}-\Psi(z)\right)^{\rho}\left(e^{\mathcal{U}} \circ \psi\right)(z) \mathcal{U}^{\prime}(\Psi(z)) d z\right] \\
& =\frac{e^{\mathcal{U}\left(d_{1}\right)}}{2}+I_{1}
\end{aligned}
$$

and

$$
\begin{aligned}
& \frac{\Gamma(\rho+1)}{2\left(d_{2}-d_{1}\right)^{\rho}} I_{d_{2}^{-}}^{\rho, \Psi}\left(e^{\mathcal{U}} \circ \Psi\right)\left(\Psi^{-1}\left(d_{1}\right)\right) \\
= & \frac{\rho}{2\left(d_{2}-d_{1}\right)^{\rho}} \int_{\Psi^{-1}\left(d_{1}\right)}^{\psi^{-1}\left(d_{2}\right)} \psi^{\prime}(z)\left(\Psi(z)-d_{1}\right)^{\rho-1}\left(e^{\mathcal{U}} \circ \Psi\right)(z) d z \\
= & \frac{1}{2\left(d_{2}-d_{1}\right)^{\rho}} \int_{\Psi^{-1}\left(d_{1}\right)}^{\psi^{-1}\left(d_{2}\right)}\left(e^{\mathcal{U}} \circ\right) d\left(\Psi(z)-d_{1}\right)^{\rho} \\
= & \frac{1}{2\left(d_{2}-d_{1}\right)^{\rho}}\left[\left(d_{2}-d_{1}\right)^{\rho} e^{\mathcal{U}\left(d_{2}\right)}-\int_{\Psi^{-1}\left(d_{1}\right)}^{\Psi^{-1}\left(d_{2}\right)} \Psi^{\prime}(z)\left(\Psi(z)-d_{1}\right)^{\rho}\left(e^{\mathcal{U}} \circ \psi\right)(z) \mathcal{U}^{\prime}(\Psi(z)) d z\right] \\
= & \frac{e^{\mathcal{U}\left(d_{2}\right)}}{2}-I_{2} .
\end{aligned}
$$

It follows that

$$
\frac{e^{\mathcal{U}}\left(d_{2}\right)+e^{\mathcal{U}}\left(d_{1}\right)}{2}-I_{1}-I_{2}=\mathbb{I} .
$$

This completes the proof. 
Theorem 2. Let $\mathcal{U}:\left[d_{1}, d_{2}\right] \rightarrow \mathcal{R}$ be a differentiable mapping, for $0 \leq d_{1}<d_{2}$, and $e^{\mathcal{U}} \in L_{1}\left(\left[d_{1}, d_{2}\right]\right)$. Let $\Psi(x)$ be an increasing and positive monotone function on $\left(d_{1}, d_{2}\right]$, with continuous derivative $\Psi^{\prime}(x)$ on $\left(d_{1}, d_{2}\right)$ and $\rho \in(0,1)$. If $\left|\mathcal{U}^{\prime}\right|^{q}$ is exponentially convex and $q \geq 1$, then

$$
\begin{aligned}
& \left|\frac{e^{\mathcal{U}\left(d_{1}\right)}+e^{\mathcal{U}\left(d_{2}\right)}}{2}-\frac{\Gamma(\rho+1)}{2\left(d_{2}-d_{1}\right)^{\rho}}\left[I_{d_{1}^{+}}^{\rho, \Psi}\left(e^{\mathcal{U}} \circ \Psi\right)\left(\Psi^{-1}\left(d_{2}\right)\right)+I_{d_{2}^{-}}^{\rho, \Psi}\left(e^{\mathcal{U}} \circ \Psi\right)\left(\Psi^{-1}\left(d_{1}\right)\right)\right]\right| \\
& \frac{d_{2}-d_{1}}{2}\left(\frac{2}{\rho+1}\left(1-\frac{1}{2^{\rho}}\right)\right)^{1-\frac{1}{q}}\left[\frac{1}{\rho+3}\left(1-\frac{1}{2^{\rho+2}}\right)\right]^{\frac{1}{q}}\left[\left|e^{\mathcal{U}\left(d_{1}\right)} \mathcal{U}^{\prime}\left(d_{1}\right)\right|^{q}+\left|e^{\mathcal{U}\left(d_{2}\right)} \mathcal{U}^{\prime}\left(d_{2}\right)\right|^{q}\right]^{\frac{1}{q}} .
\end{aligned}
$$

Proof. First note that, for every $z \in\left(\Psi^{-1}\left(d_{1}\right) \Psi^{-1}\left(d_{2}\right)\right)$, we have $d_{1}<\Psi(z)<d_{2}$. Let $\tau=\frac{d_{2}-\Psi(z)}{d_{2}-d_{1}}$, then we have $\Psi(z)=\tau d_{1}+(1-\tau) d_{2}$. Applying Lemma 1 , Hölder's inequality and exponentially convexity of $\left|\mathcal{U}^{\prime}\right|$, we obtain

$$
\begin{aligned}
& \left|\frac{e^{\mathcal{U}\left(d_{1}\right)}+e^{\mathcal{U}\left(d_{2}\right)}}{2}-\frac{\Gamma(\rho+1)}{2\left(d_{2}-d_{1}\right)^{\rho}}\left[I_{d_{1}^{\rho}}^{\rho, \Psi}\left(e^{\mathcal{U}} \circ \Psi\right)\left(\Psi^{-1}\left(d_{2}\right)\right)+I_{d_{2}^{-}}^{\rho, \Psi}\left(e^{\mathcal{U}} \circ \Psi\right)\left(\Psi^{-1}\left(d_{1}\right)\right)\right]\right| \\
& \leq \frac{1}{2}\left[\int_{\Psi-1}^{\Psi^{-1}\left(d_{1}\right)}\left|\left(\frac{\Psi(z)-d_{1}}{d_{2}-d_{1}}\right)^{\rho}-\left(\frac{d_{2}-\Psi(z)}{d_{2}-d_{1}}\right)^{\rho}\right|\left|\left(e^{\mathcal{U}} \circ \Psi\right)(z)\right| d(\mathcal{U}(\Psi(z)))\right] \\
& =\frac{d_{2}-d_{1}}{2}\left(\int_{0}^{1}\left|(1-\tau)^{\rho}-\tau^{\rho}\right| d \tau\right)^{1-\frac{1}{q}}\left(\int_{0}^{1}\left|(1-\tau)^{\rho}-\tau^{\rho}\right|\left|e^{\mathcal{U}\left(\tau d_{1}+(1-\tau) d_{2}\right)} \mathcal{U}^{\prime}\left(\tau d_{1}+(1-\tau) d_{2}\right)\right|^{q} d \tau\right)^{\frac{1}{q}} \\
& \leq \frac{d_{2}-d_{1}}{2}\left(\int_{0}^{1}\left|(1-\tau)^{\rho}-\tau^{\rho}\right| d \tau\right)^{1-\frac{1}{q}} \\
& \times\left(\int_{0}^{1}\left|(1-\tau)^{\rho}-\tau^{\rho}\right| \mid\left\{\tau\left|e^{\mathcal{U}\left(d_{1}\right)}\right|^{q}+(1-\tau)\left|e^{\mathcal{U}\left(d_{2}\right)}\right|^{q}\right\}\left\{\tau\left|\mathcal{U}^{\prime}\left(d_{1}\right)\right|^{q}+(1-\tau)\left|\mathcal{U}^{\prime}\left(d_{2}\right)\right|^{q}\right\} d \tau\right)^{\frac{1}{q}} \\
& =\frac{d_{2}-d_{1}}{2}\left(\frac{2}{\rho+1}\left(1-\frac{1}{2^{\rho}}\right)\right)^{1-\frac{1}{q}}\left(\int _ { 0 } ^ { 1 } | ( 1 - \tau ) ^ { \rho } - \tau ^ { \rho } | \left[\tau^{2}\left|e^{\mathcal{U}\left(d_{1}\right)} \mathcal{U}^{\prime}\left(d_{1}\right)\right|^{q}\right.\right. \\
& \left.\left.+(1-\tau)^{2}\left|e^{\mathcal{U}\left(d_{2}\right)} \mathcal{U}^{\prime}\left(d_{2}\right)\right|^{q}+\tau(1-\tau)\left\{\left|e^{\mathcal{U}\left(d_{1}\right)} \mathcal{U}^{\prime}\left(d_{2}\right)\right|^{q}+\left|e^{\mathcal{U}\left(d_{2}\right)} \mathcal{U}^{\prime}\left(d_{1}\right)\right|^{q}\right\}\right] d \tau\right)^{\frac{1}{q}} \\
& =\frac{d_{2}-d_{1}}{2}\left(\frac{2}{\rho+1}\left(1-\frac{1}{2^{\rho}}\right)\right)^{1-\frac{1}{q}} \\
& \times\left(\int_{0}^{1}\left|(1-\tau)^{\rho}-\tau^{\rho}\right|\left[\tau^{2}\left|e^{\mathcal{U}\left(d_{1}\right)} \mathcal{U}^{\prime}\left(d_{1}\right)\right|^{q}+(1-\tau)^{2}\left|e^{\mathcal{U}\left(d_{2}\right)} \mathcal{U}^{\prime}\left(d_{2}\right)\right|^{q}+\tau(1-\tau) \Delta\left(d_{1}, d_{2}\right)\right] d \tau\right)^{\frac{1}{q}} \\
& :=\frac{d_{2}-d_{1}}{2}\left(\frac{2}{\rho+1}\left(1-\frac{1}{2^{\rho}}\right)\right)^{1-\frac{1}{q}}\left(S_{1}+S_{2}\right) \text {. }
\end{aligned}
$$

where

$$
\begin{aligned}
& S_{1}:=\int_{0}^{\frac{1}{2}}\left[(1-\tau)^{\rho}-\tau^{\rho}\right]\left[\tau^{2}\left|e^{\mathcal{U}\left(d_{1}\right)} \mathcal{U}^{\prime}\left(d_{1}\right)\right|^{q}+(1-\tau)^{2}\left|e^{\mathcal{U}\left(d_{2}\right)} \mathcal{U}^{\prime}\left(d_{2}\right)\right|^{q}+\tau(1-\tau) \Delta\left(d_{1}, d_{2}\right)\right] d \tau, \\
& S_{2}:=\int_{\frac{1}{2}}^{1}\left[\tau^{\rho}-(1-\tau)^{\rho}\right]\left[\tau^{2}\left|e^{\mathcal{U}\left(d_{1}\right)} \mathcal{U}^{\prime}\left(d_{1}\right)\right|^{q}+(1-\tau)^{2}\left|e^{\mathcal{U}\left(d_{2}\right)} \mathcal{U}^{\prime}\left(d_{2}\right)\right|^{q}+\tau(1-\tau) \Delta\left(d_{1}, d_{2}\right)\right] d \tau .
\end{aligned}
$$


Now

$$
\begin{aligned}
& \int_{0}^{\frac{1}{2}} \tau^{2}(1-\tau)^{\rho} d \tau=\int_{\frac{1}{2}}^{1} \tau^{\rho}(1-\tau)^{2} d \tau=\beta_{\frac{1}{2}}(3, \rho+1), \\
& \int_{0}^{\frac{1}{2}} \tau^{\rho}(1-\tau)^{2} d \tau=\int_{\frac{1}{2}}^{1} \tau^{2}(1-\tau)^{\rho} d \tau=\beta_{\frac{1}{2}}(\rho+1,3), \\
& \int_{0}^{\frac{1}{2}} \tau^{\rho+2} d \tau=\int_{\frac{1}{2}}^{1}(1-\tau)^{\rho+2} d \tau=\frac{1}{(\rho+3) 2^{\rho+3}}, \\
& \int_{0}^{\frac{1}{2}}(1-\tau)^{\rho+2} d \tau=\int_{\frac{1}{2}}^{1} \tau^{\rho+2} d \tau=\frac{1}{\rho+3}-\frac{1}{(\rho+3) 2^{\rho+3}}, \\
& \int_{0}^{\frac{1}{2}}\left[(1-\tau)^{\rho+1} \tau-\tau^{\rho+1}(1-\tau)\right] d \tau=\beta_{\frac{1}{2}}(\rho+2,2)-\beta_{\frac{1}{2}}(2, \rho+2)=0, \\
& \int_{\frac{1}{2}}^{1}\left[\tau^{\rho+1}(1-\tau)-(1-\tau)^{\rho+1} \tau\right] d \tau=\beta_{\frac{1}{2}}(\rho+2,2)-\beta_{\frac{1}{2}}(2, \rho+2)=0 .
\end{aligned}
$$

By substituting the above integral values in (12) and after some simplification, we get the required inequality (13).

Corollary 1. Letting $q=1$, then under the assumption of Theorem 2, we have

$$
\begin{aligned}
& \left|\frac{e^{\mathcal{U}\left(d_{1}\right)}+e^{\mathcal{U}\left(d_{2}\right)}}{2}-\frac{\Gamma(\rho+1)}{2\left(d_{2}-d_{1}\right)^{\rho}}\left[I_{d_{1}^{+}}^{\rho, \Psi}\left(e^{\mathcal{U}} \circ \Psi\right)\left(\Psi^{-1}\left(d_{2}\right)\right)+I_{d_{2}^{-}}^{\rho, \Psi}\left(e^{\mathcal{U}} \circ \Psi\right)\left(\Psi^{-1}\left(d_{1}\right)\right)\right]\right| \\
& \leq \frac{d_{2}-d_{1}}{2}\left[\frac{1}{\rho+3}\left(1-\frac{1}{2^{\rho+2}}\right)\right]\left[\left|e^{\mathcal{U}\left(d_{1}\right)} \mathcal{U}^{\prime}\left(d_{1}\right)\right|+\left|e^{\mathcal{U}\left(d_{2}\right)} \mathcal{U}^{\prime}\left(d_{2}\right)\right|\right] .
\end{aligned}
$$

Proof. Since $\Psi$ is differentiable and strictly increasing function, we can write $(\Psi(x)-\Psi(\tau))^{\rho-1} \leq$ $\left(\Psi(x)-\Psi\left(d_{1}\right)\right)^{\rho-1}$, where as $x \in\left[d_{1}, d_{2}\right]$ and $\tau \in\left[d_{1}, x\right], \rho \geq 1$, and $\Psi^{\prime}(\tau)>0$. Then, the subsequent inequality holds true

$$
\Psi^{\prime}(\tau)(\Psi(x)-\Psi(\tau))^{\rho-1} \leq \Psi^{\prime}(\tau)\left(\Psi(x)-\Psi\left(d_{1}\right)\right)^{\rho-1}
$$

By exponentially convexity of $\mathcal{U}$, we have

$$
e^{\mathcal{U}(\tau)} \leq \frac{x-\tau}{x-d_{1}} e^{\mathcal{U}\left(d_{1}\right)}+\frac{\tau-d_{1}}{x-d_{1}} e^{\mathcal{U}(x)} .
$$

From (14) and (15), one has

$$
\begin{aligned}
& \int_{d_{1}}^{x} \Psi^{\prime}(\tau)(\Psi(x)-\Psi(\tau))^{\rho-1} e^{\mathcal{U}(\tau)} d \tau \\
\leq & \frac{\left(\Psi(x)-\Psi\left(d_{1}\right)\right)^{\rho-1}}{x-d_{1}}\left[e^{\mathcal{U}\left(d_{1}\right)} \int_{d_{1}}^{x}(x-\tau) \Psi^{\prime}(\tau) d \tau+e^{\mathcal{U}(x)} \int_{d_{1}}^{x}\left(\tau-d_{1}\right) \Psi^{\prime}(\tau) d \tau\right] .
\end{aligned}
$$


By using (2) of Definition 2, we get

$$
\begin{aligned}
& \Gamma(\rho) I_{d_{1}^{+}}^{\rho, \Psi} e^{\mathcal{U}(x)} \\
\leq & \frac{\left(\Psi(x)-\Psi\left(d_{1}\right)\right)^{\rho-1}}{x-d_{1}}\left[\left(x-d_{1}\right)\left[\Psi(x) e^{\mathcal{U}(x)}-e^{\mathcal{U}\left(d_{1}\right)} \Psi\left(d_{1}\right)\right]-\left(e^{\mathcal{U}(x)}-e^{\mathcal{U}\left(d_{1}\right)}\right) \int_{d_{1}}^{x} \Psi(\tau) d \tau\right] .
\end{aligned}
$$

Now for $x \in\left[d_{1}, d_{2}\right], \tau \in\left[x, d_{2}\right]$ and $\delta \geq 1$, the subsequent inequality holds true

$$
\Psi^{\prime}(\tau)(\Psi(\tau)-\Psi(x))^{\delta-1} \leq \Psi^{\prime}(\tau)\left(\Psi\left(d_{2}\right)-\Psi(x)\right)^{\delta-1}
$$

By exponentially convexity of $\mathcal{U}$, we have

$$
e^{\mathcal{U}(\tau)} \leq \frac{\tau-x}{d_{2}-x} e^{\mathcal{U}\left(d_{2}\right)}+\frac{d_{2}-\tau}{d_{2}-x} e^{\mathcal{U}(x)}
$$

Adopting the same procedure as we did for (14) and (15), one can get from (17) and (18) the coming inequality

$$
\begin{aligned}
& \Gamma(\delta) I_{d_{2}^{-}}^{\delta, \Psi} e^{\mathcal{U}(x)} \\
\leq & \frac{\left(\Psi\left(d_{2}\right)-\Psi(x)\right)^{\delta-1}}{d_{2}-x}\left[\left(d_{2}-x\right)\left[\Psi\left(d_{2}\right) e^{\mathcal{U}\left(d_{2}\right)}-e^{\mathcal{U}(x)} \Psi(x)\right]-\left(e^{\mathcal{U}\left(d_{2}\right)}-e^{\mathcal{U}(x)}\right) \int_{x}^{d_{2}} \Psi(\tau) d \tau\right] .
\end{aligned}
$$

From inequalities (16) and (19), we get (13). Hence the proof is completed.

Particular cases are stated as follows.

Corollary 2. Choosing $\rho=\delta$ in (13), then we have a new inequality for $\mathcal{G} \mathcal{R} \mathcal{L} \mathcal{F} \mathcal{I}$;

$$
\begin{aligned}
& \Gamma(\rho) I_{d_{1}^{+}}^{\rho, \Psi} e^{\mathcal{U}(x)}+\Gamma(\rho) I_{d_{2}^{-}}^{\rho, \Psi} e^{\mathcal{U}(x)} \\
& \leq \frac{\left(\Psi(x)-\Psi\left(d_{1}\right)\right)^{\rho-1}}{x-d_{1}}\left[\left(x-d_{1}\right)\left[\Psi(x) e^{\mathcal{U}(x)}-e^{\mathcal{U}\left(d_{1}\right)} \Psi\left(d_{1}\right)\right]-\left(e^{\mathcal{U}(x)}-e^{\mathcal{U}\left(d_{1}\right)}\right) \int_{d_{1}}^{x} \Psi(\tau) d \tau\right] \\
& +\frac{\left(\Psi\left(d_{2}\right)-\Psi(x)\right)^{\rho-1}}{d_{2}-x}\left[\left(d_{2}-x\right)\left[\Psi\left(d_{2}\right) e^{\mathcal{U}\left(d_{2}\right)}-e^{\mathcal{U}(x)} \Psi(x)\right]-\left(e^{\mathcal{U}\left(d_{2}\right)}-e^{\mathcal{U}(x)}\right) \int_{x}^{d_{2}} \Psi(\tau) d \tau\right] .
\end{aligned}
$$

Corollary 3. Choosing $x=d_{1}$ and $x=d_{2}$ in (13), adding the resulting inequalities, then the conditions of Theorem 1 are satisfied, we have

$$
\begin{aligned}
& \Gamma(\rho) I_{d_{1}^{+}}^{\rho, \Psi} e^{\mathcal{U}\left(d_{2}\right)}+\Gamma(\delta) I_{d_{2}^{-}}^{\delta, \Psi} e^{\mathcal{U}\left(d_{1}\right)} \leq\left(\frac{\left(\Psi\left(d_{2}\right)-\Psi\left(d_{1}\right)\right)^{\rho-1}+\left(\Psi\left(d_{2}\right)-\Psi\left(d_{1}\right)\right)^{\delta-1}}{d_{2}-d_{1}}\right) \\
& \times\left(\left(d_{2}-d_{1}\right)\left[e^{\mathcal{U}\left(d_{2}\right)} \Psi\left(d_{2}\right)-e^{\mathcal{U}\left(d_{1}\right)} \Psi\left(d_{1}\right)\right]-\left[e^{\mathcal{U}\left(d_{2}\right)}-e^{\mathcal{U}\left(d_{1}\right)}\right] \int_{d_{1}}^{d_{2}} \Psi(\tau) d \tau\right) .
\end{aligned}
$$


Corollary 4. If we take $\rho=\delta$ in (20), then we get the following inequality for $\mathcal{G} \mathcal{R} \mathcal{L} \mathcal{F} \mathcal{I}$

$$
\begin{aligned}
& \Gamma(\rho)\left[I_{d_{1}^{+}}^{\rho+1, \Psi} \Psi\left(d_{2}\right)+I_{d_{2}^{-}}^{\rho+1, \Psi} \Psi\left(d_{1}\right)\right] \leq\left(\frac{2\left(\Psi\left(d_{2}\right)-\Psi\left(d_{1}\right)\right)^{\rho-1}}{d_{2}-d_{1}}\right) \\
& \times\left(\left(d_{2}-d_{1}\right)\left[e^{\mathcal{U}\left(d_{2}\right)} \Psi\left(d_{2}\right)-e^{\mathcal{U}\left(d_{1}\right)} \Psi\left(d_{1}\right)\right]-\left[e^{\mathcal{U}\left(d_{2}\right)}-e^{\mathcal{U}\left(d_{1}\right)}\right] \int_{d_{1}}^{d_{2}} \Psi(\tau) d \tau\right) .
\end{aligned}
$$

Theorem 3. Let $\mathcal{U}, \Psi:\left[d_{1}, d_{2}\right] \longrightarrow \mathcal{R}$ be the functions such that $e^{\mathcal{U}}$ be differentiable function, $\Psi$ is also differentiable and strictly increasing with $\Psi^{\prime} \in L_{1}\left(\left[d_{1}, d_{2}\right]\right)$. Then for $\rho, \delta>0, x \in\left[d_{1}, d_{2}\right]$ we have

$$
\begin{aligned}
& \left|\Gamma(\rho+1) I_{d_{1}^{+}}^{\rho, \Psi} e^{\mathcal{U}(x)}+\Gamma(\delta+1) I_{d_{2}^{+}}^{\delta, \Psi} e^{\mathcal{U}(x)}-\left(\Psi(x)-\Psi\left(d_{1}\right)\right)^{\rho} e^{\mathcal{U}\left(d_{1}\right)}+\left(\Psi\left(d_{2}\right)-\Psi(x)\right)^{\delta} e^{\mathcal{U}\left(d_{2}\right)}\right| \\
\leq & \frac{\left(\Psi(x)-\Psi\left(d_{1}\right)\right)^{\rho}\left(x-d_{1}\right)\left|e^{\mathcal{U}\left(d_{1}\right)} \mathcal{U}^{\prime}\left(d_{1}\right)\right|+\left(\Psi\left(d_{2}\right)-\Psi(x)\right)^{\delta}\left(d_{2}-x\right) \mid e^{\mathcal{U}\left(d_{2}\right) \mathcal{U}^{\prime}\left(d_{2}\right) \mid}}{3} \\
& +\frac{\left\{\left(\Psi\left(d_{2}\right)-\Psi(x)\right)^{\delta}\left(d_{2}-x\right)+\left(\Psi(x)-\Psi\left(d_{1}\right)\right)^{\rho}\left(x-d_{1}\right)\right\}\left|e^{\mathcal{U}(x)} \mathcal{U}^{\prime}(x)\right|}{3} \\
& +\frac{\left(\Psi(x)-\Psi\left(d_{1}\right)\right)^{\rho}\left(x-d_{1}\right) \Delta\left(d_{1}, x\right)+\left(\Psi\left(d_{2}\right)-\Psi(x)\right)^{\delta}\left(d_{2}-x\right) \Delta\left(d_{2}, x\right)}{6}
\end{aligned}
$$

where

$$
\begin{aligned}
& \Delta\left(d_{1}, x\right)=\left|e^{\mathcal{U}(x)} \mathcal{U}^{\prime}\left(d_{1}\right)\right|+\left|e^{\mathcal{U}\left(d_{1}\right)} \mathcal{U}^{\prime}(x)\right| \\
& \Delta\left(d_{2}, x\right)=\left|e^{\mathcal{U}(x)} \mathcal{U}^{\prime}\left(d_{2}\right)\right|+\left|e^{\mathcal{U}\left(d_{2}\right)} \mathcal{U}^{\prime}(x)\right|
\end{aligned}
$$

respectively.

Proof. From the convexity of $\left|\left(e^{\mathcal{U}}\right)^{\prime}\right|$, we obtain

$$
\begin{aligned}
\left|e^{\mathcal{U}(\tau)} \mathcal{U}^{\prime}(\tau)\right| \leq & {\left[\frac{x-\tau}{x-d_{1}}\left|e^{\mathcal{U}\left(d_{1}\right)}\right|+\frac{\tau-d_{1}}{x-d_{1}}\left|e^{\mathcal{U}(x}\right|\right]\left[\frac{x-\tau}{x-d_{1}}\left|\mathcal{U}^{\prime}\left(d_{1}\right)\right|+\frac{\tau-d_{1}}{x-d_{1}}\left|\mathcal{U}^{\prime}(x)\right|\right] } \\
\leq & \left(\frac{x-\tau}{x-d_{1}}\right)^{2}\left|e^{\mathcal{U}\left(d_{1}\right)} \mathcal{U}^{\prime}\left(d_{1}\right)\right|+\left(\frac{\tau-d_{1}}{x-d_{1}}\right)^{2}\left|e^{\mathcal{U}(x)} \mathcal{U}^{\prime}(x)\right| \\
& +\left(\frac{x-\tau}{x-d_{1}}\right)\left(\frac{\tau-d_{1}}{x-d_{1}}\right)\left\{\left|e^{\mathcal{U}\left(d_{1}\right)} \mathcal{U}^{\prime}(x)\right|+\left|e^{\mathcal{U}(x)} \mathcal{U}^{\prime}\left(d_{1}\right)\right|\right\} \\
= & \left(\frac{x-\tau}{x-d_{1}}\right)^{2}\left|e^{\mathcal{U}\left(d_{1}\right)} \mathcal{U}^{\prime}\left(d_{1}\right)\right|+\left(\frac{\tau-d_{1}}{x-d_{1}}\right)^{2}\left|e^{\mathcal{U}(x)} \mathcal{U}^{\prime}(x)\right|+\left(\frac{x-\tau}{x-d_{1}}\right)\left(\frac{\tau-d_{1}}{x-d_{1}}\right) \Delta\left(d_{1}, x\right) .
\end{aligned}
$$

From (24), we have

$$
e^{\mathcal{U}(\tau)} \mathcal{U}^{\prime}(\tau) \leq\left(\frac{x-\tau}{x-d_{1}}\right)^{2}\left|e^{\mathcal{U}\left(d_{1}\right)} \mathcal{U}^{\prime}\left(d_{1}\right)\right|+\left(\frac{\tau-d_{1}}{x-d_{1}}\right)^{2}\left|e^{\mathcal{U}(x)} \mathcal{U}^{\prime}(x)\right|+\left(\frac{x-\tau}{x-d_{1}}\right)\left(\frac{\tau-d_{1}}{x-d_{1}}\right) \Delta\left(d_{1}, x\right) .
$$

Since $\Psi$ is a differentiable and strictly increasing function, we have the subsequent inequality

$$
(\Psi(x)-\Psi(\tau))^{\rho} \leq\left(\Psi\left((x)-\Psi\left(d_{1}\right)\right)^{\rho},\right.
$$

where as $x \in\left[d_{1}, d_{2}\right]$ and $\tau \in\left[d_{1}, x\right], \rho>0$.

From (25) and (26), one has 


$$
\begin{aligned}
& (\Psi(x)-\Psi(\tau))^{\rho} e^{\mathcal{U}(\tau)} \mathcal{U}^{\prime}(\tau) \\
\leq & \frac{\left(\Psi(x)-\Psi\left(d_{1}\right)\right)^{\rho}}{\left(x-d_{1}\right)^{2}}\left[(x-\tau)^{2}\left|e^{\mathcal{U}\left(d_{1}\right)} \mathcal{U}^{\prime}\left(d_{1}\right)\right|+\left(\tau-d_{1}\right)^{2}\left|e^{\mathcal{U}(x)} \mathcal{U}^{\prime}(x)\right|+(x-\tau)\left(\tau-d_{1}\right) \Delta\left(d_{1}, x\right)\right] .
\end{aligned}
$$

Integrating over $\left[d_{1}, x\right]$, we have

$$
\begin{aligned}
& \int_{d_{1}}^{x}(\Psi(x)-\Psi(\tau))^{\rho} e^{\mathcal{U}(\tau)} \mathcal{U}^{\prime}(\tau) d \tau \\
\leq & \frac{\left(\Psi(x)-\Psi\left(d_{1}\right)\right)^{\rho}}{\left(x-d_{1}\right)^{2}}\left[\left|e^{\mathcal{U}\left(d_{1}\right)} \mathcal{U}^{\prime}\left(d_{1}\right)\right| \int_{d_{1}}^{x}(x-\tau)^{2} d \tau+\left|e^{\mathcal{U}(x)} \mathcal{U}^{\prime}(x)\right| \int_{d_{1}}^{x}\left(\tau-d_{1}\right)^{2} d \tau+\Delta\left(d_{1}, x\right) \int_{d_{1}}^{x}(x-\tau)\left(\tau-d_{1}\right) d \tau\right] \\
= & \left(\Psi(x)-\Psi\left(d_{1}\right)\right)^{\rho}\left(x-d_{1}\right)\left[\frac{2\left\{\left|e^{\mathcal{U}\left(d_{1}\right)} \mathcal{U}^{\prime}\left(d_{1}\right)\right|+\left|e^{\mathcal{U}(x)} \mathcal{U}^{\prime}(x)\right|\right\}+\Delta\left(d_{1}, x\right)}{6}\right],
\end{aligned}
$$

and

$$
\begin{aligned}
& \int_{d_{1}}^{x}(\Psi(x)-\Psi(\tau))^{\rho} e^{\mathcal{U}(\tau)} \mathcal{U}^{\prime}(\tau) d \tau=\left.e^{\mathcal{U}(\tau)}(\Psi(x)-\Psi(\tau))^{\rho}\right|_{d_{1}} ^{x}+\rho \int_{d_{1}}^{x}(\Psi(x)-\Psi(\tau))^{\rho-1} e^{\mathcal{U}(\tau)} \Psi^{\prime}(\tau) d \tau \\
= & -e^{\mathcal{U}\left(d_{1}\right)}\left(\Psi(x)-\Psi\left(d_{1}\right)\right)^{\rho}+\Gamma(\rho+1) I_{d_{1}^{+}}^{\rho \Psi} e^{\mathcal{U}(x) .}
\end{aligned}
$$

Therefore (28) takes the form

$$
\begin{aligned}
& \Gamma(\rho+1) I_{d_{1}^{+}}^{\rho, \Psi} e^{\mathcal{U}(x)}-e^{\mathcal{U}\left(d_{1}\right)}\left(\Psi(x)-\Psi\left(d_{1}\right)\right)^{\rho} \\
\leq & \left(\Psi(x)-\Psi\left(d_{1}\right)\right)^{\rho}\left(x-d_{1}\right)\left[\frac{2\left\{\left|e^{\mathcal{U}\left(d_{1}\right)} \mathcal{U}^{\prime}\left(d_{1}\right)\right|+\left|e^{\mathcal{U}(x)} \mathcal{U}^{\prime}(x)\right|\right\}+\Delta\left(d_{1}, x\right)}{6}\right]
\end{aligned}
$$

Also from (24), one has

$$
e^{\mathcal{U}(\tau)} \mathcal{U}^{\prime}(\tau) \geq-\left[\left(\frac{x-\tau}{x-d_{1}}\right)^{2}\left|e^{\mathcal{U}\left(d_{1}\right)} \mathcal{U}^{\prime}\left(d_{1}\right)\right|+\left(\frac{\tau-d_{1}}{x-d_{1}}\right)^{2}\left|e^{\mathcal{U}(x)} \mathcal{U}^{\prime}(x)\right|+\left(\frac{x-\tau}{x-d_{1}}\right)\left(\frac{\tau-d_{1}}{x-d_{1}}\right) \Delta\left(d_{1}, x\right)\right] .
$$

Following the same procedure as we did for (25), we also have

$$
\begin{aligned}
& e^{\mathcal{U}\left(d_{1}\right)}\left(\Psi(x)-\Psi\left(d_{1}\right)\right)^{\rho}-\Gamma(\rho+1) I_{d_{1}^{+}}^{\rho, \Psi} e^{\mathcal{U}(x)} \\
\leq & \left(\Psi(x)-\Psi\left(d_{1}\right)\right)^{\rho}\left(x-d_{1}\right)\left[\frac{2\left\{\left|e^{\mathcal{U}\left(d_{1}\right)} \mathcal{U}^{\prime}\left(d_{1}\right)\right|+\left|e^{\mathcal{U}(x)} \mathcal{U}^{\prime}(x)\right|\right\}+\Delta\left(d_{1}, x\right)}{6}\right]
\end{aligned}
$$

From (29) and (31), we get

$$
\begin{aligned}
& \left|\Gamma(\rho+1) I_{d_{1}^{+}}^{\rho, \Psi} e^{\mathcal{U}(x)}-e^{\mathcal{U}\left(d_{1}\right)}\left(\Psi(x)-\Psi\left(d_{1}\right)\right)^{\rho}\right| \\
\leq & \left(\Psi(x)-\Psi\left(d_{1}\right)\right)^{\rho}\left(x-d_{1}\right)\left[\frac{2\left\{\left|e^{\mathcal{U}\left(d_{1}\right)} \mathcal{U}^{\prime}\left(d_{1}\right)\right|+\left|e^{\mathcal{U}(x)} \mathcal{U}^{\prime}(x)\right|\right\}+\Delta\left(d_{1}, x\right)}{6}\right] .
\end{aligned}
$$

By convexity of $\left|\left(e^{\mathcal{U}}\right)^{\prime}\right|$, we have

$$
\begin{aligned}
\left|e^{\mathcal{U}(\tau)} \mathcal{U}^{\prime}(\tau)\right| \leq & \left(\frac{\tau-x}{d_{2}-x}\right)^{2}\left|e^{\mathcal{U}\left(d_{2}\right)} \mathcal{U}^{\prime}\left(d_{2}\right)\right|+\left(\frac{d_{2}-\tau}{d_{2}-x}\right)^{2}\left|e^{\mathcal{U}(x)} \mathcal{U}^{\prime}(x)\right| \\
& +\left(\frac{\tau-x}{d_{2}-x}\right)\left(\frac{d_{2}-\tau}{d_{2}-x}\right) \Delta\left(d_{2}, x\right) .
\end{aligned}
$$

Now for $x \in\left[d_{1}, d_{2}\right]$ and $\tau \in\left[x, d_{2}\right]$ and $\delta>0$, the following inequality holds true 


$$
(\Psi(\tau)-\Psi(x))^{\delta} \leq\left(\Psi\left(d_{2}\right)-\Psi(x)\right)^{\delta} .
$$

Following the same way as we have done for (25), (26) and (30) we can get from (33) and (34) the subsequent inequality

$$
\begin{aligned}
& \left|\Gamma(\delta+1) I_{d_{2}^{+}}^{\delta, \Psi} e^{\mathcal{U}(x)}-e^{\mathcal{U}\left(d_{2}\right)}\left(\Psi\left(d_{2}\right)-\Psi(x)\right)^{\delta}\right| \\
\leq & \left(\Psi\left(d_{2}\right)-\Psi(x)\right)^{\delta}\left(d_{2}-x\right)\left[\frac{2\left\{\left|e^{\mathcal{U}\left(d_{2}\right)} \mathcal{U}^{\prime}\left(d_{2}\right)\right|+\left|e^{\mathcal{U}(x)} \mathcal{U}^{\prime}(x)\right|\right\}+\Delta\left(d_{2}, x\right)}{6}\right] .
\end{aligned}
$$

From inequalities (32) and (35) using triangular inequality, we get (21) which is desired.

Particular cases are stated as follows.

Corollary 5. Choosing $\rho=\delta$ in (21), then we have a new inequality for $\mathcal{G R} \mathcal{L} \mathcal{F} \mathcal{I}$

$$
\begin{aligned}
& \left|\Gamma(\rho+1)\left[I_{d_{1}^{+}}^{\rho, \Psi} e^{\mathcal{U}(x)}+I_{d_{2}^{+}}^{\rho, \Psi} e^{\mathcal{U}(x)}\right]-\left(\Psi(x)-\Psi\left(d_{1}\right)\right)^{\rho} e^{\mathcal{U}\left(d_{1}\right)}+\left(\Psi\left(d_{2}\right)-\Psi(x)\right)^{\rho} e^{\mathcal{U}\left(d_{2}\right)}\right| \\
\leq & \frac{\left(\Psi(x)-\Psi\left(d_{1}\right)\right)^{\rho}\left(x-d_{1}\right)\left|e^{\mathcal{U}\left(d_{1}\right)} \mathcal{U}^{\prime}\left(d_{1}\right)\right|+\left(\Psi\left(d_{2}\right)-\Psi(x)\right)^{\rho}\left(d_{2}-x\right)\left|e^{\mathcal{U}\left(d_{2}\right)} \mathcal{U}^{\prime}\left(d_{2}\right)\right|}{3} \\
& +\frac{\left\{\left(\Psi\left(d_{2}\right)-\Psi(x)\right)^{\rho}\left(d_{2}-x\right)+\left(\Psi(x)-\Psi\left(d_{1}\right)\right)^{\rho}\left(x-d_{1}\right)\right\}\left|e^{\mathcal{U}(x)} \mathcal{U}^{\prime}(x)\right|}{3} \\
& +\frac{\left(\Psi(x)-\Psi\left(d_{1}\right)\right)^{\rho}\left(x-d_{1}\right) \Delta\left(d_{1}, x\right)+\left(\Psi\left(d_{2}\right)-\Psi(x)\right)^{\rho}\left(d_{2}-x\right) \Delta\left(d_{2}, x\right)}{6} .
\end{aligned}
$$

To prove our next result we need the following Lemma.

Lemma 3. Suppose that $\mathcal{U}:\left[d_{1}, d_{2}\right] \rightarrow \mathcal{R}$ is an exponentially convex function which is symmetric about $\frac{d_{1}+d_{2}}{2}$. Then we have

$$
e^{\mathcal{U}\left(\frac{d_{1}+d_{2}}{2}\right)} \leq e^{\mathcal{U}(x)}, \quad x \in\left[d_{1}, d_{2}\right]
$$

Proof. Write

$$
\frac{d_{1}+d_{2}}{2}=\frac{1}{2}\left(\frac{x-d_{1}}{d_{2}-d_{1}} d_{2}+\frac{d_{2}-x}{d_{2}-d_{1}} d_{1}\right)+\frac{1}{2}\left(\frac{x-d_{1}}{d_{2}-d_{1}} d_{1}+\frac{d_{2}-x}{d_{2}-d_{1}} d_{2}\right) .
$$

Since $e^{\mathcal{U}}$ is convex, therefore we have

$$
\begin{aligned}
e^{\mathcal{U}\left(\frac{d_{1}+d_{2}}{2}\right)} & \leq \frac{1}{2} e^{\mathcal{U}\left(\frac{x-d_{1}}{d_{2}-d_{1}} d_{2}+\frac{d_{2}-x}{d_{2}-d_{1}} d_{1}\right)}+\frac{1}{2} e^{\mathcal{U}\left(\frac{x-d_{1}}{d_{2}-d_{1}} d_{1}+\frac{d_{2}-x}{d_{2}-d_{1}} d_{2}\right)} \\
& =\frac{1}{2}\left[e^{\mathcal{U}(x)}+e^{\mathcal{U}\left(d_{1}+d_{2}-x\right)}\right] .
\end{aligned}
$$

Also, $e^{\mathcal{U}}$ is symmetric about $\frac{d_{1}+d_{2}}{2}$, therefore we have $e^{\mathcal{U}(x)}=e^{\mathcal{U}\left(d_{1}+d_{2}-x\right)}$ and the inequality in (36) holds. 
Theorem 4. Suppose that $\mathcal{U}:\left[d_{1}, d_{2}\right] \longrightarrow \mathcal{R}$ be an exponentially convex function such that $e^{\mathcal{U}}$ is positive convex and symmetric about $\frac{d_{1}+d_{2}}{2}, \Psi$ is a differentiable and strictly increasing function having $\Psi^{\prime} \in L_{1}\left(\left[d_{1}, d_{2}\right]\right)$. Then $\rho, \delta>0$, we have

$$
\begin{aligned}
& e^{\mathcal{U}\left(\frac{d_{1}+d_{2}}{2}\right)}\left[\frac{\left(\Psi\left(d_{2}\right)-\Psi\left(d_{1}\right)\right)^{\rho+1}}{\rho+1}+\frac{\left(\Psi\left(d_{2}\right)-\Psi\left(d_{1}\right)\right)^{\delta+1}}{\delta+1}\right] \\
\leq \quad & \Gamma(\rho+1) I_{d_{1}^{+}}^{\rho+1, \Psi} \Psi\left(d_{2}\right)+\Gamma(\delta+1) I_{d_{2}^{-}}^{\delta+1, \Psi} \Psi\left(d_{1}\right) \leq \frac{\left(\Psi\left(d_{2}\right)-\Psi\left(d_{1}\right)\right)^{\delta}+\left(\Psi\left(d_{2}\right)-\Psi\left(d_{1}\right)\right)^{\rho}}{d_{2}-d_{1}} \\
& \times\left[\left(d_{2}-d_{1}\right)\left[e^{\mathcal{U}\left(d_{2}\right)} \Psi\left(d_{2}\right)-e^{\mathcal{U}\left(d_{1}\right)} \Psi\left(d_{1}\right)\right]-\left[e^{\mathcal{U}\left(d_{2}\right)}-e^{\mathcal{U}\left(d_{1}\right)}\right] \int_{d_{1}}^{d_{2}} \Psi(\tau) d \tau\right] .
\end{aligned}
$$

Proof. Since $\Psi$ is differentiable and strictly increasing function therefore $\left(\Psi(x)-\Psi\left(d_{1}\right)\right)^{\delta} \leq\left(\Psi\left(d_{2}\right)-\right.$ $\left.\Psi\left(d_{1}\right)\right)^{\delta}$, where as $x \in\left[d_{1}, d_{2}\right], \delta>0$, and $\Psi^{\prime}(x)>0$. Hence, the following inequality holds true

$$
\Psi^{\prime}(x)\left(\Psi(x)-\Psi\left(d_{1}\right)\right)^{\delta} \leq \Psi^{\prime}(x)\left(\Psi\left(d_{2}\right)-\Psi\left(d_{1}\right)\right)^{\delta} .
$$

From the exponential convexity of $\mathcal{U}$, it can be obtained

$$
e^{\mathcal{U}(x)} \leq \frac{x-d_{1}}{d_{2}-d_{1}} e^{\mathcal{U}\left(d_{2}\right)}+\frac{d_{2}-x}{d_{2}-d_{1}} e^{\mathcal{U}\left(d_{1}\right)} .
$$

From (38) and (39), one can have

$$
\begin{aligned}
& \int_{d_{1}}^{d_{2}} e^{\mathcal{U}(x)} \Psi^{\prime}(x)\left(\Psi(x)-\Psi\left(d_{1}\right)\right)^{\delta} d x \\
\leq & \frac{\left(\Psi\left(d_{2}\right)-\Psi\left(d_{1}\right)\right)^{\delta}}{d_{2}-d_{1}}\left[e^{\mathcal{U}\left(d_{2}\right)} \int_{d_{1}}^{d_{2}}\left(x-d_{1}\right) \Psi^{\prime}(x) d x+e^{\mathcal{U}\left(d_{1}\right)} \int_{d_{1}}^{d_{2}}\left(d_{2}-x\right) \Psi^{\prime}(x) d x\right] .
\end{aligned}
$$

By using (2) of Definition 2, we get

$$
\begin{aligned}
& \Gamma(\delta+1) I_{d_{2}^{-}}^{\delta+1, \Psi} e^{\mathcal{U}\left(d_{1}\right)} \\
\leq & \frac{\left(\Psi\left(d_{2}\right)-\Psi\left(d_{1}\right)\right)^{\delta}}{d_{2}-d_{1}}\left[\left(d_{2}-d_{1}\right)\left[e^{\mathcal{U}\left(d_{2}\right)} \Psi\left(d_{2}\right)-e^{\mathcal{U}}\left(d_{1}\right) \Psi\left(d_{1}\right)\right]-\left[e^{\mathcal{U}\left(d_{2}\right)}-e^{\mathcal{U}\left(d_{1}\right)}\right] \int_{d_{1}}^{d_{2}} \Psi(x) d x\right] .
\end{aligned}
$$

Now for $x \in\left[d_{1}, d_{2}\right], \tau \in\left[x, d_{2}\right]$ and $\rho>0$, the following inequality holds true

$$
\Psi^{\prime}(x)\left(\Psi\left(d_{2}\right)-\Psi(x)\right)^{\rho} \leq \Psi^{\prime}(x)\left(\Psi\left(d_{2}\right)-\Psi\left(d_{1}\right)\right)^{\rho} .
$$

Adopting the same procedure as we did for (38) and (39) one can get from (40) and (42) the subsequent inequality

$$
\begin{aligned}
& \Gamma(\rho+1) I_{d_{1}^{+}}^{\rho+1, \Psi} e^{\mathcal{U}\left(d_{2}\right)} \\
\leq & \frac{\left(\Psi\left(d_{2}\right)-\Psi\left(d_{1}\right)\right)^{\rho}}{d_{2}-d_{1}}\left[\left(d_{2}-d_{1}\right)\left[e^{\mathcal{U}\left(d_{2}\right)} \Psi\left(d_{2}\right)-e^{\mathcal{U}}\left(d_{1}\right) \Psi\left(d_{1}\right)\right]-\left[e^{\mathcal{U}\left(d_{2}\right)}-e^{\mathcal{U}\left(d_{1}\right)}\right] \int_{d_{1}}^{d_{2}} \Psi(x) d x\right] .
\end{aligned}
$$


From (41) and (43), we get

$$
\begin{aligned}
& \Gamma(\delta+1) I_{d_{2}^{-}}^{\delta+1, \Psi} e^{\mathcal{U}\left(d_{1}\right)}+\Gamma(\rho+1) I_{d_{1}^{+}}^{\rho+1, \Psi} e^{\mathcal{U}\left(d_{2}\right)} \leq \frac{\left(\Psi\left(d_{2}\right)-\Psi\left(d_{1}\right)\right)^{\delta}+\left(\Psi\left(d_{2}\right)-\Psi\left(d_{1}\right)\right)^{\rho}}{d_{2}-d_{1}} \\
& \times\left[\left(d_{2}-d_{1}\right)\left[e^{\mathcal{U}\left(d_{2}\right)} \Psi(b)-e^{\mathcal{U}\left(d_{1}\right)} \Psi\left(d_{1}\right)\right]-\left[e^{\mathcal{U}\left(d_{2}\right)}-e^{\mathcal{U}\left(d_{1}\right)}\right] \int_{d_{1}}^{d_{2}} \Psi(x) d x\right] .
\end{aligned}
$$
we get

Using Lemma 3 and multiplying (36) with $\left(\Psi(x)-\Psi\left(d_{1}\right)\right)^{\delta} \Psi^{\prime}(x)$ and integrating over $\left[d_{1}, d_{2}\right]$,

$$
e^{\mathcal{U}\left(\frac{d_{1}+d_{2}}{2}\right)} \int_{d_{1}}^{d_{2}}\left(\Psi(x)-\Psi\left(d_{1}\right)\right)^{\delta} \Psi^{\prime}(x) d x \leq \int_{d_{1}}^{d_{2}} e^{\mathcal{U}(x)}\left(\Psi(x)-\Psi\left(d_{1}\right)\right)^{\delta} \Psi^{\prime}(x) d x .
$$

By using (2) of Definition 2 we get

$$
e^{\mathcal{U}\left(\frac{d_{1}+d_{2}}{2}\right)}\left[\frac{\left(\Psi\left(d_{2}\right)-\Psi\left(d_{1}\right)\right)^{\delta+1}}{\delta+1}\right] \leq \Gamma(\delta+1) I_{d_{2}^{-}}^{\delta \Psi} e^{\mathcal{U}\left(d_{1}\right)} .
$$

Similarly, using Lemma 3 and multiplying (36) with $\left(\Psi\left(d_{2}\right)-\Psi(x)\right)^{\rho} \Psi^{\prime}(x)$ and integrating over $\left[d_{1}, d_{2}\right]$, we get

$$
e^{\mathcal{U}\left(\frac{d_{1}+d_{2}}{2}\right)}\left[\frac{\left(\Psi\left(d_{2}\right)-\Psi\left(d_{1}\right)\right)^{\rho+1}}{\rho+1}\right] \leq \Gamma(\rho+1) I_{d_{1}^{+}}^{\rho+1, \Psi} e^{\mathcal{U}\left(d_{2}\right)} .
$$

From (44) and (47), we get (37) which is the required result.

Corollary 6. Choosing $\rho=\delta$ in (37), then we have a new inequality for $\mathcal{G} \mathcal{R} \mathcal{L} \mathcal{F} \mathcal{I}$

$$
\begin{aligned}
& 2 \frac{\left(\Psi\left(d_{2}\right)-\Psi\left(d_{1}\right)\right)^{\rho+1}}{\rho+1} e^{\mathcal{U}\left(\frac{d_{1}+d_{2}}{2}\right)} \leq \Gamma(\rho+1)\left[I_{d_{1}^{+}}^{\rho+1, \Psi} \Psi\left(d_{2}\right)+I_{d_{2}^{-}}^{\rho+1, \Psi} \Psi\left(d_{1}\right)\right]\left(\frac{2\left(\Psi\left(d_{2}\right)-\Psi\left(d_{1}\right)\right)^{\rho-1}}{d_{2}-d_{1}}\right) \\
& \times\left(\left(d_{2}-d_{1}\right)\left[e^{\mathcal{U}\left(d_{2}\right)} \Psi\left(d_{2}\right)-e^{\mathcal{U}\left(d_{1}\right)} \Psi\left(d_{1}\right)\right]-\left[e^{\mathcal{U}\left(d_{2}\right)}-e^{\mathcal{U}\left(d_{1}\right)}\right] \int_{d_{1}}^{d_{2}} \Psi(\tau) d \tau\right) .
\end{aligned}
$$

\section{Examples}

Example 5. Let $d_{1}=2, d_{2}=4, \rho=2, \delta=2, \mathcal{U}(x)=2 \ln x, e^{\mathcal{U}(x)}=x^{2}, \Psi(x)=x^{2}$ and $x \in[2,4]$. Then all the assumptions in Theorem 1 are satisfied.

Clearly,

$$
\begin{aligned}
\Gamma(\rho) I_{d_{1}^{+}}^{\rho, \Psi} e^{\mathcal{U}(x)} & =\int_{d_{1}}^{x} \Psi^{\prime}(\tau)(\Psi(x)-\Psi(\tau))^{\rho-1} e^{\mathcal{U}(\tau)} d \tau \\
& =2 \int_{2}^{3}\left(9-\tau^{2}\right) \tau^{3} d t \approx 70.83,
\end{aligned}
$$


and

$$
\begin{aligned}
\Gamma(\delta) I_{d_{2}^{-}}^{\delta, \Psi} e^{\mathcal{U}(x)} & =\int_{x}^{d_{2}} \Psi^{\prime}(t)(\Psi(\tau)-\Psi(x))^{\rho-1} e^{\mathcal{U}(\tau)} d \tau \\
& =2 \int_{3}^{4}\left(\tau^{2}-9\right) \tau^{3} d \tau \approx 334.83 .
\end{aligned}
$$

Adding the above equations, we have the left-hand side term of (13)

$$
\Gamma(\rho) I_{d_{1}^{+}}^{\rho, \Psi} e^{\mathcal{U}(x)}+\Gamma(\delta) I_{d_{2}^{-}}^{\delta, \Psi} e^{\mathcal{U}(x)} \approx 405.66 .
$$

On the other hand,

$$
\begin{aligned}
& \frac{\left(\Psi(x)-\Psi\left(d_{1}\right)\right)^{\rho-1}}{x-d_{1}}\left[\left(x-d_{1}\right)\left[\Psi(x) e^{\mathcal{U}(x)}-e^{\mathcal{U}\left(d_{1}\right)} \Psi\left(d_{1}\right)\right]-\left(e^{\mathcal{U}(x)}-e^{\mathcal{U}\left(d_{1}\right)}\right) \int_{d_{1}}^{x} \Psi(\tau) d \tau\right] \\
& \quad+\frac{\left(\Psi\left(d_{2}\right)-\Psi(x)\right)^{\delta-1}}{d_{2}-x}\left[\left(d_{2}-x\right)\left[\Psi\left(d_{2}\right) e^{\mathcal{U}\left(d_{2}\right)}-e^{\mathcal{U}(x)} \Psi(x)\right]-\left(e^{\mathcal{U}\left(d_{2}\right)}-e^{\mathcal{U}(x)}\right) \int_{x}^{d_{2}} \Psi(\tau) d \tau\right] \\
& =\frac{(9-4)}{3-2}\left[(3-2)[9(9)-4(4)]-(9-4) \int_{2}^{3} \tau^{2} d \tau\right] \\
& \quad+\frac{(16-9)}{4-3}\left[(4-3)[16(16)-9(9)]-(16-9) \int_{3}^{4} \tau^{2} d \tau\right] \\
& =166.675+620.83 \approx 785.50 .
\end{aligned}
$$

It is nice to see that the following implications hold in (48) and (49),

$$
405.66<785.50 .
$$

Example 6. Let $d_{1}=2, d_{2}=4, \rho=2, \delta=2, \mathcal{U}(x)=2 \ln x, e^{\mathcal{U}(x)}=x^{2}, \Psi(x)=x^{2}$ and $x \in[2,4]$. where $\Delta\left(x, d_{1}\right)$ and $\Delta\left(x, d_{2}\right)$ are given in (22) and (23), respectively. Then all the assumptions in Theorem 3 are satisfied.

Clearly,

$$
\begin{aligned}
\Gamma(\rho+1) I_{d_{1}^{+}}^{\rho, \Psi} e^{\mathcal{U}(x)}-\left(\Psi(x)-\Psi\left(d_{1}\right)\right)^{\rho} e^{\mathcal{U}(a)} & =\int_{d_{1}}^{x}(\Psi(x)-\Psi(\tau))^{\rho} e^{\mathcal{U}(\tau)} \mathcal{U}^{\prime}(\tau) d \tau \\
& =2 \int_{2}^{3} \tau\left(9-\tau^{2}\right)^{2} d \tau \approx 41.67
\end{aligned}
$$

and

$$
\begin{aligned}
\Gamma(\delta+1) I_{d_{2}^{-}}^{\delta, \Psi} e^{\mathcal{U}(x)}-\left(\Psi\left(d_{2}\right)-\Psi(x)\right)^{\delta} e^{\mathcal{U}\left(d_{2}\right)} & =\int_{x}^{d_{2}}(\Psi(\tau)-\Psi(x))^{\delta} e^{\mathcal{U}(\tau)} \mathcal{U}^{\prime}(\tau) d \tau \\
& =2 \int_{3}^{4} \tau\left(\tau^{2}-9\right)^{2} d \tau \approx 114.33
\end{aligned}
$$


Adding the above equations, we get the left-hand term of (21)

$$
\begin{aligned}
& \left|\Gamma(\rho+1) I_{d_{1}^{+}}^{\rho, \Psi} e^{\mathcal{U}(x)}-\left(\Psi(x)-\Psi\left(d_{1}\right)\right)^{\rho} e^{\mathcal{U}\left(d_{1}\right)}+\Gamma(\delta+1) I_{d_{2}^{\delta}}^{\delta, \Psi} e^{\mathcal{U}(x)}-\left(\Psi\left(d_{2}\right)-\Psi(x)\right)^{\delta} e^{\mathcal{U}\left(d_{2}\right)}\right| \\
& =41.67+114.33 \approx 156 .
\end{aligned}
$$

Next,

$$
\begin{aligned}
& \frac{\left(\Psi(x)-\Psi\left(d_{1}\right)\right)^{\rho}\left(x-d_{1}\right)\left|e^{\mathcal{U}\left(d_{1}\right)} \mathcal{U}^{\prime}\left(d_{1}\right)\right|+\left(\Psi\left(d_{2}\right)-\Psi(x)\right)^{\delta}\left(d_{2}-x\right)\left|e^{\mathcal{U}\left(d_{2}\right)} \mathcal{U}^{\prime}\left(d_{2}\right)\right|}{3} \\
& =\frac{(9-4)^{2}(3-2)\left|4\left(\frac{2}{2}\right)\right|+(16-9)^{2}(4-3)\left|16\left(\frac{2}{4}\right)\right|}{3}=164, \\
& \quad \frac{\left\{\left(\Psi\left(d_{2}\right)-\Psi(x)\right)^{\delta}\left(d_{2}-x\right)+\left(\Psi(x)-\Psi\left(d_{1}\right)\right)^{\rho}\left(x-d_{1}\right)\right\}\left|e^{\mathcal{U}(x)} \mathcal{U}^{\prime}(x)\right|}{3} \\
& \quad \frac{(16-9)^{2}(4-3)+(9-4)^{2}(3-2)}{3}\left|9\left(\frac{2}{3}\right)\right|=148, \\
& \quad \frac{\left(\Psi(x)-\Psi\left(d_{1}\right)\right)^{\rho}\left(x-d_{1}\right) \Delta\left(d_{1}, x\right)+\left(\Psi\left(d_{2}\right)-\Psi(x)\right)^{\delta}\left(d_{2}-x\right) \Delta\left(d_{2}, x\right)}{6} \\
& \approx 172.47 .
\end{aligned}
$$

Adding the above equations we get the right-hand side term of (21)

$$
\begin{aligned}
& \frac{\left(\Psi(x)-\Psi\left(d_{1}\right)\right)^{\rho}\left(x-d_{1}\right)\left|e^{\mathcal{U}\left(d_{1}\right)} \mathcal{U}^{\prime}\left(d_{1}\right)\right|+\left(\Psi\left(d_{2}\right)-\Psi(x)\right)^{\delta}\left(d_{2}-x\right)\left|e^{\mathcal{U}\left(d_{2}\right)} \mathcal{U}^{\prime}\left(d_{2}\right)\right|}{3} \\
& +\frac{\left\{\left(\Psi\left(d_{2}\right)-\Psi(x)\right)^{\delta}\left(d_{2}-x\right)+\left(\Psi(x)-\Psi\left(d_{1}\right)\right)^{\rho}\left(x-d_{1}\right)\right\}\left|e^{\mathcal{U}(x)} \mathcal{U}^{\prime}(x)\right|}{3} \\
& +\frac{\left(\Psi(x)-\Psi\left(d_{1}\right)\right)^{\rho}\left(x-d_{1}\right) \Delta\left(d_{1}, x\right)+\left(\Psi\left(d_{2}\right)-\Psi(x)\right)^{\delta}\left(d_{2}-x\right) \Delta\left(d_{2}, x\right)}{6} \\
& =164+148+172.47 \approx 484.47 .
\end{aligned}
$$

It is nice to see that the following implications hold (50) and (51),

$$
156<484.47
$$

\section{Applications}

We consider the following special means for arbitrary real numbers $\mu, v, \mu \neq v$ :

$$
\begin{aligned}
\mathcal{A}(\mu, v) & =\frac{\mu+v}{2}, \quad \mu, v \in \mathcal{R}, \\
\mathcal{H}(\mu, v) & =\frac{2 \mu v}{\mu+v}, \quad \mu, v \in \mathcal{R} \backslash\{0\}, \\
\mathcal{L}(\mu, v) & =\frac{v-\mu}{\ln |v|-\ln |\mu|}, \quad|\mu| \neq|v|, \mu v \neq 0, \\
\mathcal{L}_{n}(\mu, v) & =\left[\frac{v^{n+1}-\mu^{n+1}}{(n+1)(v-\mu)}\right], \quad n \in \mathcal{Z} \backslash\{-1,0\}, \mu, v \in \mathcal{R}, \mu \neq v .
\end{aligned}
$$

Now using the results in Section 1, we have some applications to the special means of real numbers. 
Proposition 6. For $a_{1}, a_{2} \in \mathcal{R}^{+}, a_{1}<a_{2}$, then

$$
\left|\mathcal{A}\left(e^{a_{1}}, e^{a_{2}}\right)-\mathcal{L}\left(e^{a_{1}}, e^{a_{2}}\right)\right| \leq \frac{a_{2}-a_{1}}{2^{2-\frac{1}{q}}}\left(\frac{7}{32}\right)^{\frac{1}{q}}\left[\left|e^{a_{1}}\right|^{q}+\left|e^{a_{2}}\right|^{q}\right]^{\frac{1}{q}} .
$$

Proof. Apply Theorem 2 with $e^{\mathcal{U}(x)}=e^{x}, \Psi(x)=x, \rho=1$, and we obtain the desired result.

Proposition 6. For $a_{1}, a_{2} \in \mathcal{R}^{+}, a_{1}<a_{2}$, then

$$
\left|\mathcal{H}^{-1}\left(a_{1}, a_{2}\right)-\mathcal{L}^{-1}\left(a_{1}, a_{2}\right)\right| \leq \frac{a_{2}-a_{1}}{2^{2-\frac{1}{q}}}\left(\frac{7}{32}\right)^{\frac{1}{q}}\left[\left|\frac{1}{a_{1}}\right|^{2 q}+\left|\frac{1}{a_{2}}\right|^{2 q}\right]^{\frac{1}{q}} .
$$

Proof. Apply Theorem 2 with $e^{\mathcal{U}(x)}=\frac{1}{x}, \Psi(x)=x, \rho=1$, and we obtain the desired result.

Proposition 6. Let $a_{1}, a_{2} \in \mathcal{R}^{+}, a_{1}<a_{2}$, then

$$
\left|\mathcal{A}\left(a_{1}^{2}, a_{2}^{2}\right)-\mathcal{L}_{2}^{2}\left(a_{1}, a_{2}\right)\right| \leq \frac{a_{2}-a_{1}}{2^{1-\frac{1}{q}}}\left(\frac{7}{32}\right)^{\frac{1}{q}}\left[\left|a_{1}\right|^{q}+\left|a_{2}\right|^{q}\right]^{\frac{1}{q}} .
$$

Proof. Apply Theorem 2 with $e^{\mathcal{U}(x)}=x^{2}, \Psi(x)=x, \rho=1$, and we obtain the desired result.

Proposition 6. Let $a_{1}, a_{2} \in \mathcal{R}^{+}, a_{1}<a_{2}$, then

$$
\left|\mathcal{A}\left(a_{1}^{n}, a_{2}^{n}\right)-\mathcal{L}_{n}^{n}\left(a_{1}, a_{2}\right)\right| \leq \frac{n\left(a_{2}-a_{1}\right)}{2^{2-\frac{1}{q}}}\left(\frac{7}{32}\right)^{\frac{1}{q}}\left[\left|a_{1}\right|^{(n-1) q}+\left|a_{2}\right|^{(n-1) q}\right]^{\frac{1}{q}} .
$$

Proof. Apply Theorem 2 with $e^{\mathcal{U}(x)}=x^{n}, \Psi(x)=x, \rho=1$, and we obtain the general result.

\section{Conclusions}

In this article, we have investigated a few fractional integral inequalities for $\mathcal{G} \mathcal{R} \mathcal{L} \mathcal{F} \mathcal{I}$ via exponentially convexity. These inequalities have bounds of the sum of left-sided and right-sided fractional integrals and inequalities for the function, and their first derivative in absolute value is exponentially convex. Also, fractional inequalities of $\mathcal{H} \mathcal{H}$ type for a symmetric and exponentially convex function are proved. These estimates, bounds and inequalities exist for all fractional operators are stated in Remark 1 . The method followed to produce fractional inequalities is innovative and simple. It could be followed to broaden further consequences for other classes of functions related to exponentially convex functions, using convenient fractional integral operators.

Author Contributions: All authors contributed equally to the writing of this paper. All authors read and approved the final manuscript.

Funding: The second author would like to thank Prince Sultan University for funding this work through research, group Nonlinear Analysis Methods in Applied Mathematics (NAMAM) group number RG-DES-2017-01-17.

Acknowledgments: The authors are grateful to the reviewers for their valuable and constructive suggestions.

Conflicts of Interest: The authors declare no conflict of interest.

\section{References}

1. Samko, S.G.; Kilbas, A.A.; Marichev, O.I. Fractional Integrals and Derivatives: Theory and Applications; Gordon and Breach: Yverdon, Switzerland, 1993.

2. Podlubny, I. Fractional Differential Equations; Academic Press: San Diego, CA, USA, 1999.

3. Kilbas, A.; Srivastava, H.M.; Trujillo, J.J. Theory and Application of Fractional Differential Equations; North Holland Mathematics Studies: Berlin, Germany, 2006; Volume 204. 
4. Miller, K.; Ross, B. An Introduction to the Fractional Differential Equations; John Wiley and Sons Inc.: New York, NY, USA, 1993.

5. Sonin, N.Y. On differentiation with arbitrary index. Mosc. Matem. Sbornik. 1869, 6, 1-38.

6. Letnikov, A.V. Theory of differentiation with an arbitray index. Matem. Sbornik. 1868, 3, 1-66. (In Russian)

7. Laurent, H. On the Calculation of Derivatives with Any Indices. 2004, Volume 3, pp. 240-252. Available online: http:/ / www.numdam.org/item/NAM_1884_3_3_240_0/ (accessed on 25 July 2019). (In French)

8. Magin, R.L. Magin, R.L. Fractional Calculus in Bioengineering. Crit. Rev. Biomed. Eng. 2004, 32, 1-104. [CrossRef] [PubMed]

9. Hilfer, R. Applications of Fractional Calculus in Physics; Word Scientific: Singapore, 2000.

10. Srivastava, H.M.; Tomovski, Z. Fractional calculus with an integral operator containing generalized Mittag-Leffler function in the kernal. Appl. Math. Comput. 2009, 211, 198-210.

11. Tomovski, Z.; Hilfer, R.; Srivastava, H.M. Fractional and operational calculus with generalized fractional derivative operators and Mittag-Leffler function. Integral Transforms Spec. Funct. 2011, 21, 797-814. [CrossRef]

12. Belarbi, S.; Dahmani, Z. On some new fractional integral inequalities. J. Inequal. Pure Appl. Math. 2009, 10, 86.

13. Dahmani, Z. On Minkowski and Hermite-Hadamard integral inequalities via fractional integration. Ann. Funct. 2010, 1, 51-58. [CrossRef]

14. Dahmani, Z. New inequalities in fractional integrals. Int. J. Nonlinear Sci. 2010, 9, 493-497.

15. Dahmani, Z. The Riemann-Liouville operator to generate some new inequalities. Int. J. Nonlinear Sci. 2011, 12, 452-455.

16. Denton, Z.; Vatsala, A.S. Fractional integral inequalities and applications. Comput. Math. Appl. 2010, 59, 1087-1094. [CrossRef]

17. Anastassiou, G.A. Fractional Differentiation Inequalities; Springer: NewYork, NY, USA, 2009.

18. Abdeljawad, T. A Lyapunov type inequality for fractional operators with nonsingular Mittag-Leffler kernel. J. Inequal. Appl. 2017, 2017, 130. [CrossRef] [PubMed]

19. Abdeljawad, T.; Alzabut, J. The q-Fractional Analogue for Gronwall-Type Inequality. J. Funct. Spaces Appl. 2013, 2013, 543839. [CrossRef]

20. Adjabi, Y.; Jarad, F.; Abdeljawad, T. On generalized fractional operators and a Gronwall type inequality with applications. Filomat 2017, 31, 5457-5473. [CrossRef]

21. Jarad, F.; Abdeljawad, T.; Hammouch, Z. On a class of ordinary differential equations in the frame of Atangana-Baleanu fractional derivative. Chaos Solitons Fractals 2018, 117, 16-20. [CrossRef]

22. Abdeljawad, T.; Al-Mdallal, Q.M.; Hajji, M.A. Arbitrary order fractional difference operators with discrete exponential kernels and applications. Discret. Dyn. Nat. Soc. 2017, 2017, 4149320. [CrossRef]

23. Abdeljawad, T. Fractional operators with exponential kernels and a Lyapunov type inequality. Adv. Differ. Equ. 2017, 2017, 313. [CrossRef]

24. Alzabut, J.; Abdeljawad, T.; Jarad, F.; Sudsutad, W. A Gronwall inequality via the generalized proportional fractional derivative with applications. J. Inequal. Appl. 2019, 2019, 101. [CrossRef]

25. Khan, H.; Abdeljawad, T.; Tunç, C.; Alkhazzan, A.; Khan, A. Minkowski's inequality for the AB-fractional integral operator. J. Inequal. Appl. 2019, 2019, 96. [CrossRef]

26. Sarikaya, M.Z.; Set, E.; Yaldiz, H.; Başak, N. Hermite-Hadamard's inequalities for fractional integrals and related fractional inequalities, Math. Comput. Model. 2013, 57, 2403-2407. [CrossRef]

27. Chalishajar, D.; Ravichandran, C.; Dhanalakshmi, S.; Murugesu, R. Existence of fractional impulsive functional integro-differential equations in Banach spaces. Appl. Syst. Innov. 2019, 2, 18. [CrossRef]

28. Zhang, D.; Zhang, Y.; Zhang, Z.; Ahmed, N.; Zhang, Y.; Li, F.; Belic, M.R.; Xiao, M. On unveiling the link between fractional Schrödinger equation and light propogation in honeycomb lattice. Ann. Phys. 2017, 529, 1-6. [CrossRef]

29. Zhang, Y.; Zhang, H.; Belic, M.R.; Zhu, Y.; Zhang, W.; Zhang, Y.; Christodoulides, D.N.; Xiao, M. On PT symmetry in a fractional Schrödinger equation. Laser Photonics Rev. 2016, 10, 526. [CrossRef]

30. Zhang, Y.; Liu, X.; Belic, M.R.; Zhang, W.; Zhang, Y.; Xiao, M. On propogation dynamics of a light beam in a fractional Schrödinger equation. Phys. Rev. Lett. 2015, 115, 180403. [CrossRef]

31. Jarad, F.; Abdeljawad, T. Generalized fractional derivatives and Laplace transform. Discrete Contin. Dyn. Syst. 2018. [CrossRef]

32. Ameen, R.; Jarad, F.; Abdeljawad, T. Ulam stability of fractional differential equations with a generalized Caputo derivative. Filomat 2018, 32, 5265-5274. [CrossRef] 
33. Chen, H.; Katugampola, U.N. Hermite-Hadamard and Hermite-Hadamard-Fejér type inequalities for generalized fractional integrals. J. Math. Anal. Appl. 2017, 446, 1274-1291. [CrossRef]

34. Dragomir, S.S.; Gomm, I. Some Hermite-Hadamard type inequalities for functions whose exponentials are convex. Stud. Univ. Babes-Bolyai Math. 2015, 60, 527-534

35. Dragomir, S.S.; Agarwal, R.P. Two inequalities for differentiable mappings and applications to special means of real numbers and to trapezoidal formula. Appl. Math. Lett. 1998, 11, 91-95. [CrossRef]

36. Farid, G., Nazeer, W., Saleem, M. S., Mehmood. S., Kang, S. M.: Bounds of Riemann-Liouville fractional integrals in General form via convex functions and their applications. Mathematics 2018, 6, 248. [CrossRef]

37. Alirezaei, G.; Mathar, R. On exponentially concave functions and their impact in information theory. J. Inform. Theory Appl. 2018, 9, 265-274

38. Antczak, T. On $(p, r)$-invex sets and functions. J. Math. Anal. Appl. 2001, 263, 355-379. [CrossRef]

39. Pal, S.; Wong, T.K.L. On exponentially concave functions and a new information geometry.Ann. Probab. 2018, 46, 1070-1113. [CrossRef]

40. Pecaric, J.; Jaksetic, J. On exponential convexity, Euler-Radau expansions and stolarsky means, Rad Hrvat. Matematicke Znanosti 2013, 17, 81-94.

41. Jakestic, J.; Pecaric, J. Exponential convexity method. J. Convex Anal. 2012, 20, 181-187.

42. Bernstein, S.N. Sur les fonctions absolument monotones. Acta. Math. 1929, 52, 1-66. [CrossRef]

43. Jarad, F.; Ugurlu, E.; Abdeljawad, T.; Baleanu, D. On a new class of fractional operators. Adv. Differ. Equ. 2017, 2017, 247. [CrossRef]

44. Khan, T.U.; Khan, M.A. Generalized conformable fractional operators. J. Comput. Appl. Math. 2019, 346, 378-389. [CrossRef]

(C) 2019 by the authors. Licensee MDPI, Basel, Switzerland. This article is an open access article distributed under the terms and conditions of the Creative Commons Attribution (CC BY) license (http:/ / creativecommons.org/licenses/by/4.0/). 